\title{
Características de la intoxicación por metadona: a propósito de un caso de homicidio.
}

\author{
Characteristics of the methadone intoxication: report of a \\ case of homicide.
}

\section{P.M. Garamendi', L.J. Segura-Abad ${ }^{2}$, M. López-Alcaraz'1 y J. Rodríguez}

RESUMEN

En nuestro medio el uso de la metadona se halla restringido al entorno hospitalario y en el ámbito extrahospitalario en pacientes afectos de dolor crónico y sujetos afectos de trastorno por dependencia de opiáceos en el curso de terapias de sustitución. En ambos casos, el control sanitario ha de ser estricto. A pesar de ello, los casos de intoxicación por metadona no son extraños y del mismo modo la muerte por reacción adversa a esta sustancia no es desconocida en los servicios de patología forense. Se presenta un caso en el que las circunstancias del hecho sugieren que el origen de una muerte por metadona fue de tipo homicida. El fallecido, que no era consumidor habitual de metadona, presentaba signos clínicos antemortem y hallazgos de autopsia compatibles con una muerte por intoxicación por opiáceos con resultados analíticos positivos a metadona (concentración en sangre de $0,75 \mathrm{mg} / \mathrm{L}$ ) en ausencia de otros opiáceos. Se acompaña una revisión bibliográfica sobre las características generales de la intoxicación por metadona y en la misma se hace hincapié en lo excepcional de la forma homicida en las diversas series analizadas.

Palabras clave: Metadona, intoxicación, muerte, homicidio.

Cuad Med Forense 2009; 15(56):119-128

\section{ABSTRACT}

In Spain methadone administration is restricted to hospital environment and outside hospitals it is limited to patients affected of chronic pain and opiate dependence. In all cases administration of methadone is under strict medical control. Nevertheless, in our country there are cases of intoxications by methadone and even deaths associated with this opiate analogue. We report a case of a man who died because of a methadone intoxication and probably as a consequence of an homicide. This person was not a methadone user and there were clínical and pathological signs that indicated that his death was caused by opiate intoxication. Toxicological analysis in biological samples could not find opiates but methadone (blood concentration: $0.75 \mathrm{mg} / \mathrm{L})$. We also introduce a bibliographic review about general questions related to methadone intoxication and in this review it is stressed the fact that homicide is exceptional.

Key words: Methadone, intoxication, death, homicide.

Correspondencia: Dr. Pedro Manuel Garamendi González. IML de Huelva. Plaza Isabel La Católica, 9 21071. Huelva. e-mail: imanolgaramendi@gmail.com.

${ }^{1}$ Médico Forense. Servicio de Patología Forense. IML de Huelva.

2 Médico Forense. Servicio de Laboratorio de Toxicología. IAF de Madrid.

3 Médico Forense. Servicio de Clínica Médico Forense. IML de Huelva. 


\section{INTRODUCCIÓN:}

Pese a que tras su introducción en el mercado terapéutico la metadona se empezó a utilizar con la convicción de su seguridad terapéutica pronto se hizo evidente que el margen terapéutico de esta sustancia era relativamente estrecho y por ello podían darse casos de intoxicación asociadas al consumo de metadona incluso bajo la adecuada supervisión médica $[1,2]$. La incidencia de este fenómeno es variada en las diversas series publicadas [3,4].

Así, en Alemania se describen en diversos Institutos de Medicina Legal incidencias distintas: Colonia en el periodo 1989-2000, 2 casos entre 518 muertes por drogas; Bonn en el periodo 1997-200 I, I 8 casos; Hamburgo, en el periodo 1990-1997, 8 I casos de 95 I muertes por drogas. La forma de obtención de la metadona por parte de los fallecidos en este país fue también diversa. Así, en la serie de Colonia no se describe la obtención de la metadona salvo por medios legales en el curso de tratamientos de deshabituación, mientras que en la serie de Bonn se describe un porcentaje mayoritario de casos en los que la metadona es obtenida a través mercado ilícito $[5,6,7]$.

En los países bálticos, la incidencia también es muy disímil. En Dinamarca, las muertes por metadona en el periodo 1991 a 200 I constituyeron el $41 \%$ de las muertes por drogas, mientras que en Noruega alcanzaron sólo el 15\%, en Suecia el 4\% y en Finlandia no se describieron casos. En 2002, el número de pacientes en tratamiento con metadona alcanzaba los 5000 pacientes en Dinamarca, 2000 en Noruega, 775 en Suecia y 100 en Finlandia. Según las series publicadas, además, las políticas de control del tratamiento con metadona conformaron la principal causa de estas diferencias de porcentaje en cada uno de los países nórdicos, más restrictivas en Suecia y Noruega frente a Dinamarca $[8,9,10,1 \mathrm{I}]$.

En EEUU las cifras entre los distintos estados también son muy diferentes. De este modo, mientras que en New York City la mortalidad por metadona entre 1990 y 1998 se mantuvo estable en un 13,9\% de las muertes por drogas, habiéndose obtenido la metadona mayoritariamente por medios legales en el curso de tratamientos de deshabituación, en estados como Maryland la incidencia de esta causa de muerte sufrió entre 1997 y 200 I un aumento del 950\%. La incidencia más alta se observa en Baltimore donde la tasa de mortalidad por metadona llegó a ser entre 2001 y 2006 de 2 muertes por 100.000 habitantes $[12,13,14,15]$.

En el Reino Unido, en Sheffield, con una población de 531.000 habitantes, se produjeron entre 199| y 1994 I 8 muertes por metadona y en Escocia Occidental, con una población de 2,25 millones de habitantes, 56 casos entre 1991 y 200 I. En esta última serie, además, se observó, en oposición a lo mostrado en series de otros países, una disminución de la proporción de esta causa de muerte entre el periodo 1997-200 I frente al 199 | - 1996, coincidiendo con la instauración local de programas para la supervisión de las prescripciones médicas de metadona $[16,17,18]$.

En la mayoría de las series se hace hincapié en el hecho de que se ha podido observar un aumento de la proporción de muertes por metadona en relación con varias circunstancias. En primer lugar, con la falta de un adecuado control médico de la pauta farmacológica durante las primeras semanas de tratamiento con metadona (fase de inducción) que prevea la menor tolerancia a esta sustancia de los sujetos no habituados a la metadona y la existencia incompleta de fenómenos de tolerancia cruzada con opiáceos como la heroína [I]. En segundo lugar, la falta de adecuada información de quienes administran la sustancia sobre la dosis correcta en cada momento terapéutico y su estrecho margen terapéutico, que debe obligar a limitar la cantidad de metadona a administrar a algunos pacientes. De esta manera se ha podido comprobar que las campañas de educación para médicos y farmacéuticos han surtido efectos positivos sobre la incidencia de esta causa de muerte 
y del mismo modo la restricción de la administración de la metadona en unidades especiales con formación específica [19]. En tercer lugar, la tendencia en algunos lugares a dar dosis para tomar en casa demasiado elevadas, con el fin de evitar la falta de metadona los fines de semana y festivos en que los centros expendedores permanecen cerrados. Se ha podido observar como en los lugares donde esta actitud es más laxa y por parte de los servicios médicos o farmacéuticos se da a los pacientes dosis de metadona para consumirla durante incluso una semana o un mes, la incidencia de muertes accidentales y suicidas por metadona aumenta de forma significativa $[16,17,18,20]$. Finalmente, el último factor relacionado es la existencia de un mercado ilícito de metadona, que facilita la disponibilidad de la sustancia para personas no sometidas a control médico adecuado y que posiblemente desconocen las necesidades específicas de la sustancia en cada paciente. Este mercado ilícito, además, se abastece de la metadona legalmente entregada a los pacientes en lugares donde se dan importantes cantidades de la sustancia para su consumo domiciliario y donde no se toman las adecuadas medidas para supervisar que los pacientes efectivamente toman la medicación administrada [I].

Las muertes debidas a metadona se ha podido observar que se producen en una elevada proporción durante el sueño (más del 75\% de casos en la serie de Seymour et al) [ 16,17$]$, lo que resulta previsible dada la relativamente prolongada latencia entre el inicio de los síntomas y la ingesta de la sustancia. La etiología médico legal de las intoxicaciones es a menudo no bien conocida. En algunas series se indica que se trata de suicidios en un $44 \%$ de casos, accidentes en un $5 \%$ de casos y hasta en un $47 \%$ de casos la etiología es desconocida [6]. Se describe la administración forzada de la metadona a niños en algunos casos. Suele tratarse de bebés a los que sus padres habitualmente toxicómanos administran la metadona como sedante para evitar su llanto, con un inesperado desenlace letal [2 I]. Están descritos muy pocos casos de usos de la metadona como mecanismo homicida en adultos. En la revisión realizada sólo se ha podido identificar un caso de uso forzado de metadona en un varón de 17 años en la serie de Sheffield de 1995 [2]. La dificultad para obtener datos precisos de la escena del crimen en una parte significativa de los casos puede haber sido la causa que explique esta escasa incidencia en oposición a la elevada proporción de casos de etiología no conocida.

En el presente trabajo, se presenta un caso de autopsia médico legal en el que se pudo comprobar que la causa de la muerte fue una intoxicación por metadona y que, tras la investigación del lugar de los hechos y la investigación policial, la etiología médico legal de la misma podría haber sido homicida.

\section{PRESENTACIÓN DEL CASO:}

Varón de 27 años de edad al que se le practica autopsia judicial. Entre los hallazgos de autopsia destacan: hongo de espuma, cianosis cervicofacial, pulmones de 947 y 913 gramos con signos de edema pulmonar agudo, congestión visceral generalizada y corazón de 470 gramos con signos de pericarditis fibrosa antigua y adherencias pericárdicas. El estudio histopatológico indica:

- Corazón: Pericarditis curada con fibrosis de ambas hojas pericárdicas. Arterioesclerosis coronaria leve. Congestión miocárdica.

- Timo sin alteraciones.

- Pulmones: Congestión vascular y edema pulmonares, hemorragia alveolar aguda. Antracosis pulmonar.

- Hígado y bazo: Congestión vascular aguda.

- Páncreas: Hemorragia intersticial aguda. 
Se realiza en la sala de autopsias test de detección rápida en orina mediante "5 Test Panel" de Biomerica Inc. para cocaína, anfetamina, metanfetamina, THC y opiáceos, siendo el resultado positivo para todos los tóxicos salvo opiáceos (Imagen I). Este test de detección rápida en orina identifica como opiáceos a sustancias como heroína, morfina o codeína, pero no a análogos opiáceos como la metadona.

El estudio toxicológico realizado sobre muestras viscerales en el INTyCF de Sevilla fue positivo a Cocaína, benzoilecgonia y metilecgonina, MDMA,

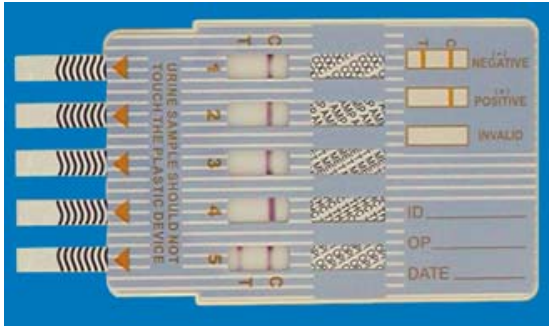

Fig. 1. Test de detección de drogas "5 Test Panel" de Biomerica Inc para cocaína, anfetamina, metanfetamina, THC y opiáceos (líneas 1 a 4). Resultado negativo para opiáceos en orina (línea 5).

Alprazolam, $\alpha$-Hidroxialprazolam, Fenacetina, Zolpidem y Metadona y EDDP. Los resultados se resumen en la tabla I. La interpretación del laboratorio para las concentraciones de metadona es la de que la misma en sangre era equivalente a la observada en sujetos normalmente sometidos a tratamiento de deshabituación de opiáceos con metadona.

\begin{tabular}{|c|c|c|c|c|c|c|c|c|c|c|}
\hline & Metadona & $\begin{array}{c}\text { EDDP } \\
(*)\end{array}$ & Cocaína & Benzoilecgonina & Metilecgonina & MDMA & Alprazolam & $\begin{array}{c}\alpha \text {-Hidroxi- } \\
\text { alprazolam }\end{array}$ & $\begin{array}{c}\text { Fenacetina } \\
(* *)\end{array}$ & $\begin{array}{c}\text { Zolpidem } \\
(* *)\end{array}$ \\
\hline Sangre & 0,75 & - & - & 0,60 & - & - & - & - & 0,37 & - \\
\hline $\begin{array}{c}\text { Humor } \\
\text { vítreo }\end{array}$ & 0,23 & Trazas & - & - & - & - & - & - & 0,27 & - \\
\hline Orina & 7,94 & Trazas & 0,06 & 360,32 & 33,56 & 17,36 & 0,09 & 0,11 & 1,88 & - \\
\hline $\begin{array}{c}\text { Contenido } \\
\text { Gástrico }\end{array}$ & 33,61 & trazas & - & - & - & - & - & - & 0,66 & 5,87 \\
\hline $\begin{array}{c}\text { Hisopo } \\
\text { nasal }\end{array}$ & - & - & + & - & - & - & - & - & + & - \\
\hline
\end{tabular}

Tabla 1. Resultados de los análisis toxicológicos practicados por el INTyCF .

* EDDP: 2-etil-1,5-difenilpirrolidina, metabolito de la metadona

** Fenacetina: analgésico con gran potencial tóxico nefrotóxico y metahemoglobinizante, así como cancerigeno, de venta no autorizada en España y que se utiliza como adulterante habitual de la cocaína

*** Zolpidem: hipnótico leve con nombres comerciales de Stilnox® y Zolpidem®

La investigación del lugar de los hechos indica que durante el levantamiento el fallecido fue identificado y según fuentes de la policía y familiares se trataba de un consumidor habitual de drogas de abuso (cocaína, cannabis, éxtasis líquido y alcohol) que no mantenía tratamiento alguno por este abuso. Tampoco constaban a estas fuentes consumo ocasional o habitual de heroína, metadona u otros opiáceos. No constan otros antecedentes médicos de interés.

Según se refirió por parte de los testigos, el fallecido había consumido dos "rayas de cocaína" y una ampolla de "MDA" líquido durante la noche del día $I^{\circ}$. Hacia las 5 horas del $2^{\circ}$ toma una copa (ginebra con coca-cola) que le da otra persona, en presencia de testigos que conocían a ambos, quien entrega y quien recibe la copa, y 10 minutos después sufre un cuadro de malestar y es trasladado a un centro sanitario. La persona que le entregó la bebida es alguien con quien el fallecido mantiene una conflictiva relación interpersonal en la que mediaron mutuas agresiones e intentos de homicidio con causas judiciales pendientes por ello. Es atendido hacia las 5. I 5 horas del día $2^{\circ}$ con un cuadro catalogado por el médico de guardia como "letargia y dificultad respiratoria con glucemia de 217 ". Se le inyecta cóctel de dos ampollas de Anexate ${ }^{\circledR}$ y dos de Naloxona, se le pasan 500 cc de suero fisiológico y ventimax a 12 lpm y tras ello, "el paciente se recupera por completo". Se le recomienda traslado a un hospital, pero el paciente pide el alta voluntaria hacia las 6 horas del día $2^{\circ}$. Tras ello, se va a casa de una amiga a dormir, quien lo encuentra cadáver hacia las 16.00 horas del mismo día $2^{\circ}$. 
No se tomaron muestras de sangre $u$ otros fluidos biológicos del fallecido durante la asistencia médica practicada el día $2^{\circ}$ hacia las 5.15 horas. Una vez hallado cadáver no se realizaron maniobras de reanimación cardiopulmonar básica o avanzada sobre el fallecido.

Paralelamente a todo ello, otra persona, amiga del fallecido, había bebido también de la misma copa que había tomado el fallecido hacia las 5.00 horas, si bien refiere haber tomado solo algunos tragos de la misma. Esta mujer también se siente mal y es trasladada a un hospital, donde ingresa a las 8.33 horas en coma con pupilas en miosis. Es tratada con Naloxona y Anexate ${ }^{\circledR}$ y se recupera, permaneciendo en observación. El electrocardiograma es de 120 ppm sin alteraciones significativas. Se realizan estudios de hemograma, bioquímica y toxicología. La impresión diagnóstica es de intoxicación por metadona, con análisis de orina negativo a Metanfetamina, Opiáceos, Cocaína y metabolitos, cannabinoides, fenciclidina, benzodiacepinas, barbitúricos, antidepresivos tricíclicos y anfetamina y positivo a metadona. La paciente refiere haber consumido cannabinoides antes del ingreso y alcohol, pero no otras sustancias y niega otros antecedentes somáticos de interés. Refiere que bebió la copa de un amigo que le supo muy raro y que posteriormente se encontró mal, perdió el conocimiento y fue llevada al centro de salud. Describe el sabor de la bebida como "a hierro".

Se realiza estudio toxicológico en el INTyCF sobre muestras de sangre y orina tomadas de esta mujer ingresada, en los que se identifican:

- Alcohol etílico: en sangre, concentración de 0,84 g/L.

- Metadona: en sangre $(0,12 \mathrm{mg} / \mathrm{L})$ y en orina $(2,53 \mathrm{mg} / \mathrm{L})$

- EDDP (metabolito de metadona): en orina $(0,07 \mathrm{mg} / \mathrm{L})$

No consta la hora en la que fueron recogidas estas muestras.

\section{DISCUSIÓN:}

La metadona es una droga de síntesis derivada de la difenilpropilamina que si bien no pertenece al grupo de los opiáceos por su estructura química, sí que comparte con estos propiedades farmacológicas al actuar como agonista casi puro de los receptores opioides tipo mu [I]. Es una sustancia que en estado natural tiene un sabor acre y amargo, por lo que habitualmente se mezcla con sustancias azucaradas para facilitar su ingesta. La droga, utilizada desde hace varios decenios en todo el mundo como base del tratamiento sustitutivo de opiáceos en terapias de deshabituación, también se utiliza en tratamientos analgésicos bajo la supervisión de las unidades del dolor [I]. La sustancia se puede obtener en presentaciones orales bajo la forma de solución líquida o comprimidos y en presentaciones para administración parenteral. En nuestro país, la droga es de uso restringido y su administración está limitada al uso hospitalario, en unidades del dolor y en centros de tratamiento de drogodependientes bajo estricto control sanitario.

La metadona se presenta en dos formas isoméricas: dextrógira y levógira. La mayor parte de la acción opiácea es mediada por la forma L, si bien las presentaciones habituales suelen llevar una proporción variable de ambas formas [I].

El inicio de los síntomas se produce entre 0,5 a I hora tras la ingesta oral. Su pico máximo de concentración lo alcanza hacia 2 a 4 horas y tiene una vida media que alcanza las 20 o 30 horas. Su biodisponibilidad oscila entre un $46 \%$ y $99 \%$, según los autores. Por su elevada liposolubilidad, la metadona se distribuye poco después de su absorción hasta en un $98 \%$ por los tejidos y solo un $2 \%$ permanece en sangre, con una tasa de unión a proteínas superior al $80 \%$. El fenómeno de inducción enzimática del metabolismo de la metadona suele demorarse entre 2 y 3 semanas desde el inicio del tratamiento, por lo que el metabolismo de la sustancia es más lento en sujetos 
no habituados en los que no se ha producido este fenómeno de inducción. Su metabolismo hepático está mediado fundamentalmente por el citocromo P-450-3A4 (CYP3A4), el 2D6 y el IA2, dando como metabolitos por N-demetilación el EDDP (2-etil- I,5-difenilpirrolidina) y el EMDP (2etil-5-metil-3,3-difenilpirrolidina), así como metadol y normetadol, aunque en menor medida. Su eliminación se realiza hasta en un 60\% (media del 25\%) por orina en las primeras 24 horas y el resto en 96 horas, en una proporción de EDDP del 42\% y de metadona en un 58\%. El resto es eliminado por heces y bilis $[1,19,22]$.

Existen evidencias de que hay variedades genéticas del sistema CYP2D6 que hacen que algunos sujetos tengan una capacidad metabólica acelerada de la metadona y otros disminuida con independencia del fenómeno de inducción por la tolerancia. Además, sustancias que son capaces de interferir, sobre todo, con el sistema 3A4 pueden alterar el metabolismo de la metadona acelerándolo o retrasándolo. En la tabla 2 se incluye una lista de algunas de las sustancias inductoras y bloqueadoras del metabolismo de la metadona. Es importante valorar estas interacciones en caso de posible intoxicación ya que el uso concomitante de rifampicina, por ejemplo, puede obligar a duplicar la dosis de metadona para evitar síndromes de abstinencia o el uso de antirretrovirales como el efavirenz puede hacer disminuir los niveles de metadona entre un 20 y $80 \%$. Por el contrario, el uso de Ketoconazol o fluoxetina puede inhibir sustancialmente el metabolismo de la metadona $[1,19,22,23,24]$.

\begin{tabular}{|c|c|}
\hline \multicolumn{2}{|c|}{ CYP3A4 } \\
\hline $\begin{array}{l}\text { Inductores } \\
\text { Barbitúricos } \\
\text { Carbamacepina } \\
\text { Dexametasona } \\
\text { Efavirenz } \\
\text { Nelfinavir } \\
\text { Nevirapina } \\
\text { Fenitoína } \\
\text { Rifampicina } \\
\text { Risperidona } \\
\text { Ritonavir } \\
\text { Topiramato }\end{array}$ & $\begin{array}{l}\text { Inhibidores } \\
\text { Cimetidina } \\
\text { Ciprofloxacino } \\
\text { Claritromicina } \\
\text { Diltiazem } \\
\text { Eritromicina } \\
\text { Fluconazol } \\
\text { Fluoxetina } \\
\text { Fluvoxamina } \\
\text { Ketoconazol } \\
\text { Norfloxacino } \\
\text { Norfluoxetina } \\
\text { Paroxetina } \\
\text { Venlafaxina } \\
\text { Zumo de uva }\end{array}$ \\
\hline \multicolumn{2}{|c|}{ CYP2A6 } \\
\hline $\begin{array}{l}\text { Inductores } \\
\text { (No puede ser inducida) }\end{array}$ & $\begin{array}{l}\text { Inhibidores } \\
\text { Cimetidina } \\
\text { Clomipramina } \\
\text { Fluoxetina } \\
\text { Fluvoxamina } \\
\text { Haloperidol } \\
\text { Levomepromazina } \\
\text { Norfluoxetina } \\
\text { Paroxetina } \\
\text { Quinidina } \\
\text { Sertralina } \\
\text { Tioridazina }\end{array}$ \\
\hline
\end{tabular}

Tabla 2. Lista de sustancias inductoras e inhibidoras de los sistemas enzimáticos CYP2D6 y CYP3A4 para la metadona. 
Además de interacciones toxicocinéticas mediadas por interferencias en el metabolismo, parece que algunas sustancias podrían disminuir el umbral de toxicidad de la metadona por interacciones sinérgicas en sus efectos sobre la depresión central y respiratoria, como el alcohol, los barbitúricos, los IMAO, las fenotiacinas o algunos tranquilizantes como las benzodiacepinas [22].

El aclaramiento de metadona por orina también se ve alterado por el $\mathrm{pH}$, de modo que la acidificación de la orina puede aumentar hasta en un 30\% la cantidad de metadona excretada por orina [24]. Del mismo modo, parece que el consumo concomitante de cocaína puede aumentar la excreción de metadona por esta misma vía, disminuyendo los niveles plasmáticos de la metadona [I].

En el tratamiento de pacientes en terapia de deshabituación de opiáceos habitualmente se recomienda que se inicie, sobre todo si no existen evidencias de una fuerte dependencia a los opiáceos, con dosis de 10 a 20 mg, nunca mayor de $30 \mathrm{mg}$, y pasar tras la fase de inducción a dosis de mantenimiento oscilantes entre 25 y $40 \mathrm{mg}$ diarios. De otro modo, es posible que si se inicia la terapia con dosis superiores a las recomendadas se puedan producir fenómenos de intoxicación por metadona bien porque el sujeto no tenga suficiente tolerancia a los opiáceos o porque dicha tolerancia no sea completamente cruzada con la metadona .

La concentración de metadona en sangre en el fallecido $(0,75 \mathrm{mg} / \mathrm{L})$ corresponde aproximadamente con las concentraciones que en la literatura consultada se describen como las habituales en sangre de pacientes que se hallan en tratamiento con dicha droga con el fin de realizar una deshabituación a opiáceos como la heroína fundamentalmente. Dichos pacientes presentan una especial tolerancia a los efectos tóxicos de los opiáceos. Sin embargo, sujetos no habituados al consumo de opiáceos o que no se hallan en tratamiento con metadona suelen tener una menor tolerancia a los efectos tóxicos de los opiáceos en general y de la metadona en particular [1, 19,22].

La intoxicación por metadona tiene, básicamente, dos niveles [25]:

- Intoxicación leve a moderada: caracterizada por letargia asociada con flaccidez muscular, disminución de la presión arterial y el pulso y del ritmo intestinal y respiratorio y miosis pupilar.

- Intoxicación grave: coma que se asocia con severa depresión respiratoria y apnea. Provoca un edema agudo pulmonar no cardiogénico, según algunos autores, también asociado a la RCP y la administración de naloxona u otros antagonistas opiáceos.

La causa de la muerte está mediada por tres tipos de efectos [1,25]:

- Patología respiratoria: fundamentalmente, el edema agudo de pulmón no cardiogénico. Sin embargo, también se asocia con otros problemas, como la aspiración de vómito por depresión central y bronconeumonía. En casos sobre todo de intoxicaciones mixtas con benzodiacepinas se asocian también alteraciones funcionales por obstrucción de vías respiratorias superiores y reacciones de tipo asmático.

- Problemas cardiacos: la metadona es un bloqueante de la conducción cardiaca. En casos de intoxicación por metadona se han evidenciado episodios de bradicardia y taquicardia y se cree que, al igual que otros análogos de la metadona como el LAAM, puede tener efecto arritmogénico. Existen evidencias de la capacidad de la metadona in vivo de producir arritmias tipo torsadas de puntas [14, I5,26,27,28,29].

- Fracaso renal: en la mayoría de casos se debe a rabdomiolisis secundarias al coma y la inmovilización prolongada.

El tratamiento básico es mediante el uso de antídotos específicos antagonistas opiáceos. El más utilizado es la naloxona, que consigue revertir todos los síntomas de la intoxicación. Sin embargo, su vida media de acción es de apenas I a 2 horas, muy inferior a la de la metadona, por lo que 
en caso de sospecha de intoxicación por metadona es necesario esperar al menos 3 a 4 horas tras la administración para asegurarse de que los síntomas de intoxicación no reaparecen. En general se recomienda el ingreso en observación y la aplicación de dosis repetidas de naloxona, si son necesarias, durante al menos 6 a 12 horas $[1,25]$.

En la literatura médica se hace hincapié en el hecho de que no se puede decir con precisión cual es la dosis mortal de metadona [1,24]. Varios factores coadyuvan a esta afirmación. De una parte, la metadona sufre fenómenos de redistribución en vida y postmortales dependientes de su amplia distribución por el componente tisular que limitan la capacidad para correlacionar con precisión las concentraciones en sangre de la droga y la cantidad total de metadona presente en el organismo. Además, la interacción tanto farmacodinámica como farmacocinética con otras drogas también constituye un obstáculo para definir con precisión el posible efecto de una determinada concentración o cantidad de droga en el organismo [19,22,30,3।].

No obstante, la mayor parte de autores consultados entienden que concentraciones en sangre superiores a $0,75 \mathrm{mg} / \mathrm{L}$ pueden resultar mortales en todo tipo de sujetos, habituados y no habituados al consumo de opiáceos. En algunas series, se han encontrado concentraciones medias en casos de intoxicación mortal por metadona que oscilan entre 0,77 y l,37 mg/L. Sin embargo, en sujetos no habituados al consumo de opiáceos o de metadona esta concentración mortal llega a ser casi la mitad (mediana entre 0,22 y 0,43 mg/L) [1,19,22]. En niños las concentraciones consideradas mortales son aún menores [2I].

En términos de dosis mortal, se considera que en sujetos no habituados la dosis mortal puede ser de apenas $50 \mathrm{mg}$ para adultos y de apenas $10 \mathrm{mg}$ para niños. Otros autores consideran, sin embargo, que estas dosis pueden incluso llegar a ser menores en sujetos no habituados.

Por las características toxicológicas de la metadona, resulta imposible estimar con precisión la dosis total de la sustancia ingerida por un sujeto dado, ya que las concentraciones plasmáticas no se correlacionan bien con la dosis ingerida. Por ello, en este caso no fue posible responder con seguridad a la cuestión de cual fue la dosis total ingerida por el fallecido y por la superviviente [24].

En ambos casos, la mujer superviviente y el fallecido, existen datos que sugieren que en el mismo periodo de tiempo (unos 30 minutos a 2 horas tras la ingesta de alguna sustancia) sufrieron una intoxicación que, por las características clínicas descritas en la asistencia médica recibida por ambos y por su respuesta a la naloxona, antagonista opiáceo específico, fue de tipo opiáceo y provocada por metadona.

De los datos obtenidos de la mujer, no es posible estimar salvo que junto con dicha intoxicación por metadona existió un consumo concomitante de alcohol etílico que pudo potenciar los efectos letárgicos.

De los datos obtenidos del fallecido, se desprende la existencia de consumo de cocaína en algún momento y posiblemente cortada con Fenacetina por vía nasal, consumo reciente de metadona por vía oral, consumo no reciente de cocaína, consumo no reciente de MDMA y consumo muy reciente de Zolpidem por vía oral. Cabe considerar que aunque hay evidencias de consumo de otras sustancias (cocaína, MDMA y Zolpidem) es improbable que dichas sustancias hayan tenido una interferencia en la intoxicación por metadona, en un caso por que su consumo era no reciente y en el otro por ser demasiado reciente para poder llegar a alcanzar una concentración suficiente en sangre.

Por otra parte, los hallazgos en los análisis toxicológicos de muestras provenientes de la mujer y el fallecido no son absolutamente congruentes con las referencias de los testigos. Así, pese a que se refiere el consumo habitual de "extasis líquido" (nombre común del ácido 
gammahidroxibutírico o GBH) y el consumo reciente antemortem de "MDA líquido" (3,4-metilendioxianfetamina, también conocido como "píldora del amor") por parte del fallecido, los hallazgos indican únicamente el consumo de MDMA (3,3.metilendioximetilanfetamina, también conocido como "éxtasis"). Del mismo modo, en la mujer se refiere el consumo de cannabis, si bien no su cantidad, y los hallazgos indican que no se pudo identificar el tetrahidrocannabinol o sus metabolitos por encima del punto de corte umbral del laboratorio. Estas incongruencias no son extrañas si se toma en consideración el valor relativo de las referencias de los testigos en el contexto médicolegal, que pueden ser altamente orientativas pero que deben ser interpretadas a la luz de otros hallazgos objetivos dentro de la investigación.

Las concentraciones de metadona en la sangre del fallecido $(0,75 \mathrm{mg} / \mathrm{L})$ son similares a las observadas en sujetos habituados al consumo de metadona tras la fase de inducción (al menos tres semanas tras el inicio del tratamiento, periodo a partir del cual se administran las dosis mayores de metadona). No obstante, según los datos bibliográficos manejados, dicha concentración se puede considerar letal en sujetos adultos no habituados a dicho consumo o al consumo de opiáceos (heroinómanos), cual era el caso en el fallecido.

Los hallazgos macroscópicos y microscópicos de la autopsia y el estudio histopatológico complementario realizado por el INTyCF de Sevilla son congruentes con el citado mecanismo de muerte por intoxicación por metadona. A este mismo nivel macroscópico y microscópico no se hallaron signos indicativos de la existencia de otras patologías que hubiesen podido provocar la muerte por sí mismas o por agravación de los efectos tóxicos de la metadona.

En ambos casos, hombre y mujer, la historia clínica sugiere la existencia antes del ingreso de una intoxicación por opiáceos que solo puede ser revertida con naloxona. Los análisis confirman posteriormente el origen de la intoxicación de la metadona. Conocida la prolongada vida media de la metadona, de 20 a 30 horas, en comparación con la de la naloxona de I a 2 horas, es lógico suponer que los efectos tóxicos de la metadona se reiniciaron una vez pasados los efectos antagonistas de la naloxona, posiblemente durante el sueño.

La etiología médico legal del fallecimiento en este caso parece haber sido homicida, lo que tratándose de un sujeto adulto resulta una circunstancia excepcional. Sin duda, en este caso, la labor de investigación del lugar de los hechos por parte del médico forense de guardia que asistió a la escena de los hechos y de los miembros de la Policía Judicial fue el factor clave para poder identificar la etiología médico legal homicida de la intoxicación.

Pese a que en España existe una supervisión de las pautas de administración de metadona por parte de servicios clínicos especializados (los CPD, Centros de Planificación de Drogas, en el caso de Andalucía), es una realidad que en nuestro país existe un mercado ilícito de metadona que facilita la posibilidad de intoxicaciones y muertes accidentales motivadas por el desconocimiento de la toxicología de esta sustancia. No cabe duda, además, de que el conocimiento de las características tóxicas y letalidad de la metadona y su disponibilidad en este mercado ilícito pueden también facilitar un eventual uso voluntario de la misma con fines tóxicos diversos y aún mortales, de forma similar a como lo fue en el caso descrito en este trabajo.

\section{AGRADECIMIENTOS:}

A los miembros de Policía Judicial de Valverde del Camino (Huelva) por su excelente trabajo de investigación de la escena de los hechos.

A los Servicios de Histopatología y Toxicología del INTyCF de Sevilla por su celeridad en la práctica de los análisis solicitados. 


\section{BIBLIOGRAFÍA:}

I. Karch SB. Pathology of drug abuse. 4th edition. CRC Press.2009

2. Clarck JC, Milroy CM, Forrest ARW. Deaths from methadone use. J Clin Forensic Med. 1995;2:143-144

3. Darke S, Duflou J. Toxicology and circumstances of death of homicide victims in New South Wales, Australia 1996-2005. J Forensic Sci.2008;53(2). DOI: 10.IIII/j.I556-4029.2008.0679.x

4. Zorec Karlovsek M. Illegal drug-related fatalities in Slovenia. Forensic Sci Int.2004;1265:S7I-S75.

5. Musshoff $F$, Lachenmeier DW, Madea B. Methadone substitution: medicolegal problems in Germany. Forensic Sci Int.2003;133:II8-I24. 6. Heinemann A, Iwersen-Bergmann S, Stein S, Schmoldt A, Püschel K. Methadone related fatalities in Hamburg 1990-1999: implications for quality standards in maintenance treatment?. Forensic Sci Int.2000;II3:449-455.

7. Grass H, Behnsen S, Kimont HG, Staak M, Käferstein H. Methadone and its role in drug related fatalities in Cologne 19892000. Forensic Sci Int.2003;132:195-200.

8. Jönsson A, Holmgren P, Ahlner J. Fatal intoxications in a Swedish forensic autopsy material during 1992-2002. Forensic Sci Int.2004;143:53-59

9. Steenfoft A, Teige B, Ceder G, Vuori E, Kristinsson J, Simonsen KW, Holmgren P, Wethe G, Kaa E. Fatal poisoning in drug addicts in the Nordic countries. Forensis Sci Int.200I;123:63-69.

10. Steenfoft A, Teige B, Holmgren P, Vuori E, Kristinsson J, Kaa E, Wethe G, Ceder G, Pikkarainen J, Simonsen KW. Fatal poisonings in young drug addicts in the Nordic countries: a comparison between 1984-1985 and 1991. Forensic Sci int.1996;78:29-37.

II. Steenfoft A, Teige B, Holmgren P, Vuori E, Kristinsson J, Hansen $A C$, Ceder $G$, Wethe $G$, Rollmann D. Fatal posioninig in drug addicts in 2002. Forensic Sci Int.2006;160:148-156.

I2. Shields LBE, Hunsaker JC, Corey TS, Ward MK, Stewart D. Methadone toxicology fatalities: a review of Medical Examiner cases in a large Metropolitan Area. J Forensic Sci.2007;52(6):1389-1395. 13. Bryant WK, Galea S, Tracy M, Piper M, Tardiff KJ, Valhov D. Overdose deaths attributed to methadone and heroin in New York City, 1990-1998. Addiction.2004;99:846-854

14. Fingerhut IA. Increases in Poisoning and Methadone-Related Deaths: United States, 1999-2005. Health E-Stat. Feb. 2008.

http://www.cdc.gov/nchs/products/pubs/pubd/hestats/poisoning/p oisoning.htm

15. Center for Substance Abuse Treatment, Methadone-Associated Mortality: Report of a National Assessment, May 8-9, 2003. SAMHSA Publication No. 04-3904. Rockville, MD: Center for Substance Abuse Treatment, Substance Abuse and Mental Health Services Administration, 2004.
16. Seymour A, Black M, Jay J, Cooper G, Weir C, Oliver J. The role of methadone in drug related deaths in the West of Scotland. Addiction.2003;98:995-1002.

17. Seymour A, Black M, Oliver JS. Drug related deaths in the Strathclyde region of Scotland, 1995-1998. Forensic Sci Int.2001;122:52-59

18. Cairns A, Roberts IS, Benbow EW. Characteristics of fatal methadone overdose in Manchester 1985-1994. Br Med J.1996;313(7052):264-265.

19. Wolff K. Characterization of methadone overdose: clinical considerations and the scientific evidence. Ther Drug Monitor.2002;24(4):457-470

20. Milroy CM, Forrest ARW. Methadone deaths: a toxicological analysis. J Clin Pathol.2000;53:277-28I

2I. Kintz P, Villain M, Dumestre-Toulet V, Capolaghi B, Cirimele V. Methadone as a chemical weapon : two fatal cases involving babies. Ther Drug Monitor.2005;27:74I-743.

22. Corkery JM, Schifano F, Ghodse AH, Oyefeso A. The effects of methadone and its role in fatalities. Human Psychopharmacol Clin Exp.2004;19:565-576.

23. Mozayani A, Raymon L. Handbook of drug interactions. A clinical and forensic guide. Humana Press. 2004

24. Segura L. Curso de formación continuada en Toxicología Forense. Toxicocinética de la metadona. Valencia 20-22 febrero 2008.

25. Olson KR. Poisoning and drug overdose. Lange Medical Books \& McGraw Hill. 2004.

26. Sánchez-Hernández AM, Atienza F, Arenal A et al. Torsade de pointes durante el tratamiento con metadona. Rev Esp Cardiol.2005;58(10):1230-2

27. Ehret GB, Voide C, Gex-Fabry M, Chabert J, Shah D, Broers B, Piguet V, Musset T, Gaspoz JM, Perrier A, Dayer P, Desmeules JA. Drug induced long QT syndrome in injection drug users receiving methadone: high frequency in hospitalized patients and risk factors. Arch Intern Med, 2006:26:1280-7

28. Fanoe S, Hvidt C, Ege P, Jensen GB. Syncope and QT prolongation among patients treated with metadone in Copenhagen. Heart 2007; 93(9):1051-5

29. Hussain T, Ewer AK. Maternal methadone may cause arrhythmias in neonates. Acta Paediatr 2007,96:768-9

30. Drummer $\mathrm{OH}$. Postmortem toxicology. Forensic Sci Int.2007;165:199-203

31. Mussoff F, Padosch S, Steinborn S, Madea B. Fatal blood and tissue concentrations of more than 200 drugs. Forensic Sci Int.2004;142:161-210. 